Ulrich Seiffert | Gotthard Rainer (Hrsg.)

Virtuelle Produktentstehung für Fahrzeug und Antrieb im Kfz 
Ulrich Seiffert | Gotthard Rainer (Hrsg.)

Virtuelle

Produktentstehung

für Fahrzeug

und Antrieb im Kfz

Prozesse, Komponenten, Beispiele aus der Praxis

Mit 359 Abbildungen

PRAXIS | ATZ/MTZ-Fachbuch

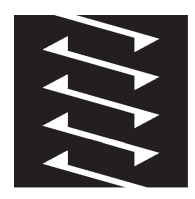


Bibliografische Information Der Deutschen Nationalbibliothek

Die Deutsche Nationalbibliothek verzeichnet diese Publikation in der

Deutschen Nationalbibliografie; detaillierte bibliografische Daten sind im Internet über

$<$ http://dnb.d-nb.de> abrufbar.

\section{Auflage 2008}

Alle Rechte vorbehalten

(C) Vieweg+Teubner Verlag | GWV Fachverlage GmbH, Wiesbaden 2008

Lektorat: Thomas Zipsner | Elisabeth Lange

Der Vieweg+Teubner Verlag ist ein Unternehmen von Springer Science+Business Media. www.viewegteubner.de

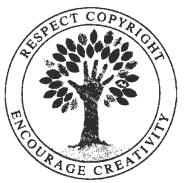

Das Werk einschließlich aller seiner Teile ist urheberrechtlich geschützt. Jede Verwertung außerhalb der engen Grenzen des Urheberrechtsgesetzes ist ohne Zustimmung des Verlags unzulässig und strafbar. Das gilt insbesondere für Vervielfältigungen, Übersetzungen, Mikroverfilmungen und die Einspeicherung und Verarbeitung in elektronischen Systemen.

Die Wiedergabe von Gebrauchsnamen, Handelsnamen, Warenbezeichnungen usw. in diesem Werk berechtigt auch ohne besondere Kennzeichnung nicht zu der Annahme, dass solche Namen im Sinne der Warenzeichen- und Markenschutz-Gesetzgebung als frei zu betrachten wären und daher von jedermann benutzt werden dürften.

Technische Redaktion: Klementz publishing services, Gundelfingen Umschlaggestaltung: KünkelLopka Medienentwicklung, Heidelberg Druck und buchbinderische Verarbeitung: MercedesDruck, Berlin Gedruckt auf säurefreiem und chlorfrei gebleichtem Papier.

Printed in Germany

ISBN 978-3-8348-0345-0 


\section{Vorwort}

Die Fahrzeugentwicklung steht nach wie vor unter steigenden Anforderungen sowohl von Seiten des Marktes als auch bedingt durch Auflagen des Gesetzgebers. Hohe Fahrzeugsicherheit und Qualitätsansprüche, geringst mögliche Abgaswerte, niedriges Außengeräusch, geringer Verbrauch und damit verbunden geringe $\mathrm{CO}_{2}$-Emissionen, akzeptable Fahrleistungen, erweiterte Variantenzahl pro Hersteller, Integration in Verkehrssysteme und internationaler Wettbewerb sind nur einige Stichwörter. Neben den Produkten sind es die Prozesse, die die Erhöhung der Wettbewerbsfähigkeit ganz entscheidend beeinflussen. In den hier zusammengestellten Beiträgen zur virtuellen Produktentstehung, die im Wesentlichen auf Präsentationen der ATZ/MTZ-Konferenzen zur VVC (Virtual Vehicle Creation) und VPC (Virtual Powertrain Creation) aufbauen, wird für das Gesamtfahrzeug aber auch für sämtliche Hauptgruppen wie Antrieb, Aufbau Fahrwerk und Elektronik durch die entsprechenden fachlichen Stellungnahmen diese veränderte Welt beschrieben. Obwohl es immer noch der physikalischen Tests auf der Basis von Hardwarekomponenten und Prototypen bedarf, trägt der veränderte Prozess nicht nur zur Erhöhung der Entwicklungsqualität sondern auch zur Verringerung der Entwicklungszeit bei. Das vorliegende Fachbuch zeigt ganzheitlich die Lösungen anhand von Praxisbeispielen auf.

Dieses Fachbuch richtet sich vor allem an in Praxis und Wissenschaft tätige Fachleute der Kfz-, Zulieferer- und Elektronikindustrie sowie an Hard- und Softwarehersteller. Auch Professoren und Studenten der Kfz-Technik bietet es einen hilfreichen Begleiter und zuverlässigen Ratgeber.

Unser besonderer Dank gilt allen unseren Autoren für ihren großen Einsatz bei der Realisierung dieses Fachbuches. Dabei sind vor allem die ausgeprägte Fachkompetenz und die verständliche Darstellung der Lösungen hervorzuheben.

Dem Vieweg Verlag, insbesondere dem Lektor Herrn Thomas Zipsner, danken wir für die jederzeit kollegiale und vorausschauende Mitarbeit. 


\section{Autorenverzeichnis}

Prof. Dr.-Ing. Dr. h.c. Albert Albers

[5.1]

Dr. Klaus U. Baron

[2.3]

Dr. rer. nat. Jost Bernasch

[6.2]

Dr.-Ing. Ernst Beutner

[1.2]

Dr.-Ing. Thomas Binder

$$
\text { [1.1] }
$$

Dipl.-Ing. Thomas Bock

[1.1]

Dr. Bernd Bohr

[2.1]

Dr.-Ing. Stefan Bunzel

$$
\text { [2.2] }
$$

Dr. Dipl.-Ing. Klaus Denkmayr

$$
\text { [3.4] }
$$

Dipl.-Ing. Arnulf Deschler

[4]

Dipl.-Ing. Michael Dick

[1.1]

Dr. Raimund Ellinger

$$
\text { [3.1] }
$$

Dipl.-Ing. Andreas Ennemoser

[5.3]

Dipl.-Ing. Helmut Fennel

[2.2]

Dr. Robert Fischer

[3.1]

Ronald Gneiting

[6.1]
Institut für Produktentwicklung,

Universität Karlsruhe

www.ipek.uni-karlsruhe.de

Delphi Deutschland GmbH, Wuppertal www.delphi.com

Kompetenzzentrum -

Das Virtuelle Fahrzeug

Forschungsgesellschaft mbH, A-Graz

www.virtuellesfahrzeug.at

IAV GmbH, Chemnitz

www.iav.de

Audi AG, Ingolstadt

www.audi.de

Audi AG, Ingolstadt

www.audi.de

Robert Bosch GmbH, Stuttgart

www.bosch.de

Continental, Frankfurt a.M.,

www.continental-corporation.com

AVL List GmbH, A-Graz

www.avl.com

ZF Friedrichshafen AG, Friedrichshafen www.zf.com

Audi AG, Ingolstadt

www.audi.de

AVL List GmbH, A-Graz

www.avl.com

AVL List GmbH, A-Graz

www.avl.com

Continental, Frankfurt a. M.,

www.continental-corporation.com

AVL List GmbH, A-Graz

www.avl.com

Behr GmbH \& Co. KG, Stuttgart

www.behrgroup.com 
Dipl.-Ing. Michael Günther

[3.6]

Dr.-Ing. Thomas Heckenberger

[6.1]

M. Sc. Borislav Klarin

[5.4]

Dr.-Ing. Peter A. Klumpp

[3.2]

Dipl.-Ing. Thomas Kriegel, MBA

[1.1]

Dipl.-Ing. Markus Kühl

[5.1]

Dr.-Ing. Gerhard Maas

[1.2]

Dr.-Ing. Franz Maaßen

[3.3]

Hinrich Meinheit

[5.2]

Dipl.-Ing. Thomas Müller

[1.1]

Prof. Dr.-Ing. Klaus Müller-Glaser

[5.1]

Dr. Peter Nefischer

[5.3]

Prof. Dr.-Ing. Horst Oehlschlaeger

[1.3]

Dipl.-Ing. Sascha Ott

[5.1]

Dr. Heinz Petutschnig

[5.3]

Prof. Dr.-Ing. Stefan Pischinger

[3.3]

Dipl.-Ing. Dr. techn. Wolfgang Puntigam [6.2]

Dr. Gotthard Rainer

[Hrsg.]
IAV GmbH, Chemnitz

www.iav.de

Behr GmbH \& Co. KG, Stuttgart

www.behrgroup.com

AVL-AST d.o.o., Zagreb, Croatia

www.avl.com

Audi AG, Ingolstadt

www.audi.com

Audi AG, Ingolstadt

www.audi.de

aquintos $\mathrm{GmbH}$, Karlsruhe

www.aquintos.com

IAV GmbH, Chemnitz

www.iav.de

FEV Motorentechnik GmbH, Aachen

www.fev.com

Institut für Kraftfahrwesen, RWTH Aachen

www.ika.rwth-aachen.de

Audi AG, Ingolstadt

www.audi.de

Institut für Technik der Informationsverarbeitung, Universität Karlsruhe www.itiv.uni-karlsruhe.de

BMW Motoren GmbH, A-Steyr

www.bmw-werk-steyr.at

Volkswagen Nutzfahrzeuge, Wolfsburg www.vwn.de

Institut für Produktentwicklung,

Universität Karlsruhe

www.ipek.uni-karlsruhe.de

AVL List GmbH, A-Graz

www.avl.com

FEV Motorentechnik GmbH, Aachen www.fev.com

Kompetenzzentrum -

Das Virtuelle Fahrzeug

Forschungsgesellschaft mbH, A-Graz

www.virtuellesfahrzeug.at

AVL List GmbH, A-Graz

www.avl.com 
Dr.-Ing. Martin Rebbert

[3.5]

Dipl.-Ing. Thomas Resch

[5.4]

Dr. Johannes-Jörg Rüger

[2.1]

Dr. Christof Samhaber

[5.3]

Dipl.-Ing. Thomas Schaffner

[5.4]

Dr.-Ing. Christian Schyr

[5.1]

Prof. Dr.-Ing. Ulrich Seiffert

[Hrsg.]

Dipl.-Ing. Martin Sopouch

[5.4]

Dr.-Ing. Karin Spors

[1.4]

Dr.-Ing. Ulrich Uphoff

[1.5]

Dipl.-Ing. Christian Vock

[5.4]

Univ.-Prof. Dr.-Ing. Henning Wallentowitz [5.2]

Dr.-Ing. Thomas Wetzel

[6.1]

Dr.-Ing. Steffen Zwahr

[3.6]
FEV Motorentechnik GmbH, Aachen www.fev.com

AVL List GmbH, A-Graz

www.avl.com

Robert Bosch GmbH, Stuttgart

www.bosch.de

BMW Motoren GmbH, A-Steyr

www.bmw-werk-steyr.at

CD Labor für Motor- und Fahrzeugakustik, A-Graz

AVL List GmbH, A-Graz

www.avl.com

WiTech Engineering GmbH, Braunschweig www.witech-engineering.de

AVL List GmbH, A-Graz

www.avl.com

Volkswagen AG, Wolfsburg

www.volkswagen.de

BMW Group München

www.bmwgroup.com

AVL List GmbH, A-Graz

www.avl.com

Institut für Kraftfahrwesen, RWTH Aachen www.ika.rwth-aachen.de

Behr GmbH \& Co. KG, Stuttgart

www.behrgroup.com

Westsächsische Hochschule, Zwickau

www.fh-zwickau.de 


\section{Inhaltsverzeichnis}

1 Gesamtfahrzeug

1.1 Einsatz virtueller Techniken in der Produktentwicklung ................................ 1

1.1.1 Einflüsse auf die Automobilindustrie .................................................. 1

1.1.1.1 Globale Trends .................................................................. 1

1.1.1.2 Anforderungen aus geänderten Kundenerwartungen ............. 4

1.1.1.3 Neue Wege gehen ............................................................... 6

1.1.2 Der Produktprozess und die Rolle der virtuellen Entwicklung …….... 7

1.1.2.1 Paradigmenwechsel im Produktprozess ………………….... 7

1.1.2.2 Synchronisation des Entwicklungsprozesses ......................... 8

1.1.2.3 Datenlogistik ........................................................................ 9

1.1.2.4 Simulation als Prozessintegrator ........................................... 12

1.1.3 Die Verwendung der virtuellen Techniken zur Komponenten- und

Eigenschaftsentwicklung …………................................................ 15

1.1.3.1 Eigenschaftsableitung ........................................................... 15

1.1.3.2 Eigenschaftsverfolgung (-Monitoring) …………………....... 17

1.1.3.3 Interdisziplinäre Prozessketten im Entwicklungsprozess ....... 18

1.1.3.4 Neue Herausforderungen in der Simulation durch Regelsysteme 20

1.1.3.4.1 Entwicklung von Fahrerassistenzsystemen ........... 21

1.1.3.4.2 Vehicle in the Loop (VIL) .................................... 22

1.1.4 Zusammenfassung und Ausblick .......................................................... 27

1.1.4.1 Geänderter Produktprozess .................................................. 27

1.1.4.2 Grenzen der virtuellen Entwicklung …………..................... 28

1.1.4.3 Ausblick ......................................................................... 29

1.2 Auslegungstools und Expertenwissen .......................................................... 29

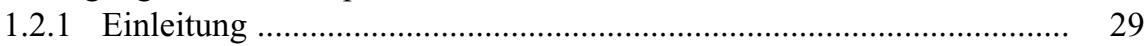

1.2.2 Der „Integrierte Entwicklungsablauf“................................................ 31

1.2.3 Mit Auslegungstools zur simulationsgestützten Entwicklung in frühen Entwicklungsphasen .................................................................. 35

1.2.4 Verstärkte Nutzung von Wissen im Engineeringprozess ..................... 42

1.2.4.1 Wissen - auf dem Weg zur Ressource .................................. 42

1.2.4.2 Lösungsansätze für wissensbasiertes Engineering ................. 44

1.2.4.3 Wissensdatenbanken ........................................................... 46

1.2.5 Anwendung von Wissensdatenbanken ............................................ $\quad 50$

1.2.5.1 Information ....................................................................... 50

1.2.5.2 Technisches Benchmarking .................................................. 50

1.2.5.3 Bereitstellung Expertenwissen .............................................. 53

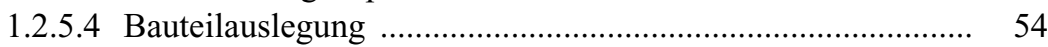

1.2.6 Zusammenfassung und Ausblick ..................................................... 56

1.3 Virtuelle Produktentwicklung in der Konzeptphase von Nutzfahrzeugen ...... 58

1.3.1 Konzeptentwicklung, Frontloading …………………………………... 59 
1.3.2 Beispiele für Simulationswerkzeuge in der Konzeptphase .................. 60

1.3.2.1 Concept Car (Parametrisches Konzeptmodell) ....................... 60

1.3.2.2 Digital Mock-Up (DMU) .................................................... 62

1.3.2.3 Virtuelle Sitzkiste ................................................................... 63

1.3.2.4 Augmented Reality ............................................................. 64

1.3.2.5 Parametrische Berechnungsverfahren .................................... 65

1.3.2.6 Modularer Berechnungsmodell-Aufbau ............................... 67

1.3.2.7 Strömungsberechnung (CFD) …………………………....... 68

1.3.2.8 Mehrkörpersimulation (MKS) .............................................. 71

1.3.2.9 Blattfedergeführte Starrachse ……………………………... 72

1.3.3 Virtuelle Produktentwicklung in der Zukunft ..................................... 73

1.4 Beschleunigung des Produktprozesses ...................................................... 75

1.4.1 Einleitung ........................................................................... 75

1.4.2 Die drei Beschleunigungskomponenten .......................................... 77

1.4.3 Ausgewählte Beispiele ..................................................................... 83

1.4.4 Zusammenfassung und Ausblick .................................................... 87

1.5 Virtueller verteilter Entwicklungsprozess bei Abgasanlagen und -konzepten 88

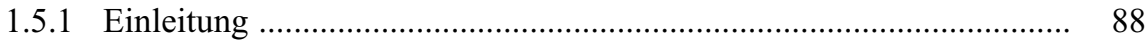

1.5.2 CAE-Methoden im Entwicklungsprozess Abgasanlage ..................... 88

1.5.3 Numerischer Werkzeugkasten für Abgasanlagenberechnungen .......... 91

1.5.3.1 Vernetzungsrichtlinie für Abgasanlagenmodelle .................. 93

1.5.3.2 Nummerierungskonvention für Abgasanlagenmodelle .......... 94

1.5.3.3 Eine hierarchisch angelegte Struktur für Abgasanlagenberechnungen ................................................ 94

1.5.3.4 Automatisierungsalgorithmus ................................................. 95

1.5.3.5 Verschiedene kleinere Programme zur Erledigung von Teilaufgaben ....................................................................... 95

1.5.4 Beispiele ………….................................................................... 95

1.5.5 Einführung einer gemeinsamen Methodik bei den Partnern ................. 97

1.5.6 Zusammenfassung ......................................................................... 98

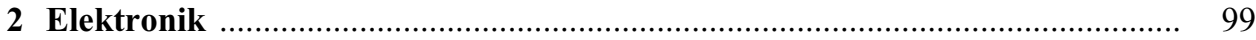

2.1 Elektronik als Schlüsseltechnologie zur unfallfreien und umweltfreundlichen Mobilität der Zukunft ................................................. 99

2.1.1 Globale Herausforderungen ………………....................................... 99

2.1.2 Elektronik und Systemvernetzung ………………............................. 101

2.1.3 Maßnahmen zur Qualitätssicherung bei Elektronik-Systemen ............ 111

2.1.4 Zusammenfassung ……………………………............................... 113

2.2 AUTOSAR - Der Standard, seine Anwendung und die weitere Entwicklung 114

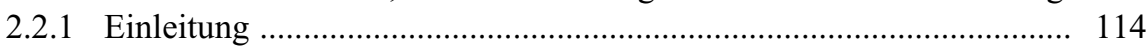

2.2.2 AUTOSAR-Projektorganisation ........................................................ 115

2.2.3 Technisches Konzept von AUTOSAR …………............................. 116

2.2.3.1 Schichtenmodell der Softwarearchitektur ............................. 116

2.2.3.2 Virtueller Funktionaler Bus .................................................... 118

2.2.3.3 Metamodell und Methodik .................................................. 120 
2.2.3.4 Konfigurationskonzept ....................................................... 122

2.2.3.5 Fehlerbehandlung .............................................................. 124

2.2.3.6 Anwendungssoftware ....................................................... 125

2.2.4 Status der AUTOSAR-Spezifikationen ………………………….... 125

2.2.4.1 AUTOSAR Release 1.0 ................................................... 125

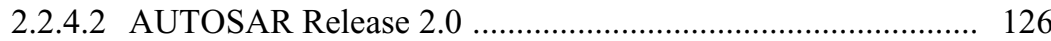

2.2.4.3 AUTOSAR Release 2.1 …............................................... 127

2.2.5 AUTOSAR Konformitätsprüfung ……………................................. 127

2.2.5.1 Zielsetzung .................................................................... 127

2.2.5.2 Prozess der Konformitätsprüfung …………………………... 128

2.2.6 AUTOSAR Phase II: 2007 - 2009 ............................................... 129

2.2.6.1 Inhalte der AUTOSAR Phase II ......................................... 129

2.2.6.2 Start von AUTOSAR Phase II ............................................ 131

2.2.7 Schlussfolgerung und Ausblick .......................................................... 131

2.3 Virtuelle Systementwicklung - Von der Anforderung zum Steuergerät ......... 133

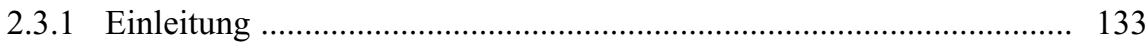

2.3.2 Anforderungsverursachte Komplexitätszunahme im

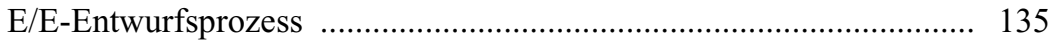

2.3.3 Konventionelle Architektur- und Steuergeräteentwicklung ……......... 138

2.3.4 Integraler Toolverbund zur virtuellen E/E-Entwicklung ..................... 140

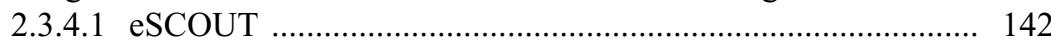

2.3.4.2 CAPEmaster/CAPEopticon ……………………................. 145

2.3.4.3 Virtuelle Hardware ............................................................. 148

2.3.4.4 HW/SW Co-Simulation ........................................................ 149

2.3.5 Schlussbetrachtung ..................................................................... 151

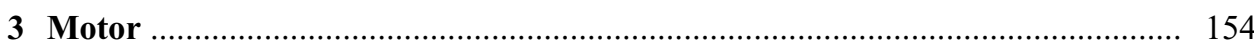

3.1 Virtuelle Antriebsstrangentwicklung …………….................................... 154

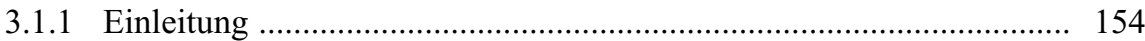

3.1.2 Entwicklungsprozess der Antriebsstrangentwicklung ……................. 155

3.1.3 Kalibrierung im Fahrzeug, auf der Straße .......................................... 156

3.1.4 Kalibrierung im Fahrzeug, auf dem Rollenprüfstand ......................... 160

3.1.5 Kalibrierung auf Motor-, Getriebe- und Antriebsstrangprüfständen .... 164

3.1.6 Kalibrierung in der Hardware-in-the-Loop (HiL) und Model-in-the-Loop (MiL) Umgebung …………………….............. 166

3.1.7 Zusammenfassung und Ausblick ..................................................... 170

3.2 Steuertriebsentwicklung mit Simulation und Versuch ................................... 172

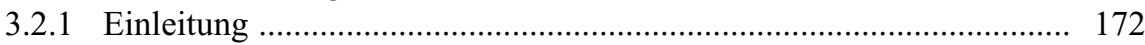

3.2.2 Entwicklungstools: Simulations- und Messtechniken ........................ 172

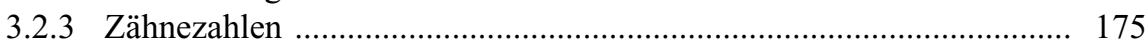

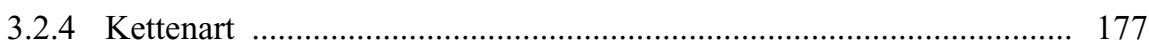

3.2.5 Dynamikergebnisse .................................................................. 180

3.2.6 Unrunde Kettenräder ................................................................. 181

3.2.7 Aufbau und Ausbau der Simulationstechnik ....................................... 184

3.2.8 Zusammenfassung ..................................................................... 188 
3.3 Virtuelle Motorenentwicklung ................................................................. 189

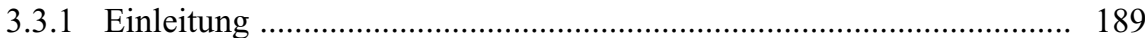

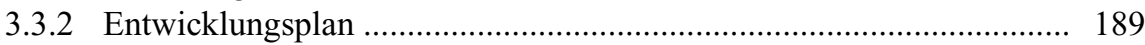

3.3.3 CAE-Unterstützung und virtuelle Produktfreigabe .............................. 191

3.3.4 Festigkeit und Kühlung ................................................................. 193

3.3.5 Ladungswechsel und Thermodynamik ............................................ 194

3.3.6 Kosten und Gewicht ....................................................................... 195

3.3.7 NVH und Gewicht .................................................................... 196

3.3.8 Dokumentation und Projektmanagement .......................................... 299

3.3.9 Motormanagement, Software und Applikation .................................. 299

3.3.10 Zusammenfassung ............................................................... 200

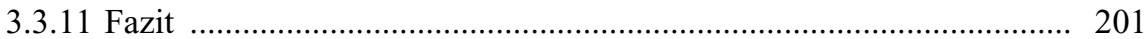

3.4 Zuverlässigkeitsmethoden in der Motorentwicklung .................................... 202

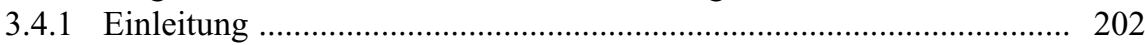

3.4.2 Der Zuverlässigkeitsprozess ………………................................ 202

3.4.2.1 Statistische Analysen ........................................................... 204

3.4.2.2 Projekt-Risikomanagement ................................................ 204

3.4.2.3 FMEA - Fehler-Möglichkeits- und Einflussanalyse .............. 205

3.4.2.4 Concern-System ............................................................... 205

3.4.2.5 Design of Experiments (DoE) und Robustheit ...................... 206

3.4.2.6 Zuverlässigkeitsblockdiagramme (Reliability Allocation) ..... 206

3.4.2.7 Intelligente Validierung - Die Load Matrix Methodik ........... 206 3.4.2.7.1 Prozess der Load Matrix Erstellung ....................... 207

3.4.2.7.2 Maßnahmen zur Verbesserung der Validierung ..... 208

3.4.2.7.3 Nutzen der Load Matrix ........................................ 208

3.4.7.8 Reliability Charts .................................................................. 209

3.4.2.9 Garantiekostenprognose .................................................... 211

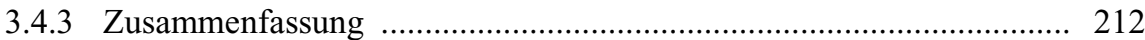

3.5 3D-Simulation der Kolbengruppe …………………................................ 213

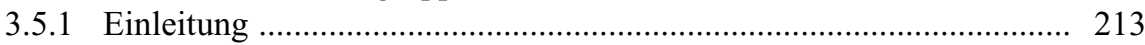

3.5.2 Simulation der Kolbengruppe in Bausteinen ...................................... 214

3.5.2.1 Thermische Strukturanalyse des Kolbens .............................. 214

3.5.2.2 Simulation der Kolbensekundärbewegung ………………..... 216

3.5.2.3 Simulation der Kolbenringdynamik ...................................... 220

3.5.3 Anwendungsbeispiel: Reibungsanalyse ........................................... 223

3.5.4 Zusammenfassung .......................................................................... 225

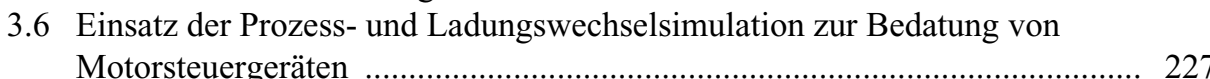

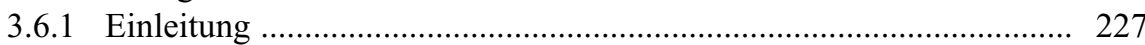

3.6.2 Motivation .............................................................................. 227

3.6.3 Grundlagen zur Einbeziehung der Vorausberechnung in den Applikationsprozess ………………………………….................. 228

3.6.4 Werkzeuge zur effektiven Grundbedatung mit Vorausberechnung ..... 230

3.6.5 Anwendung für Füllungserfassung, Momentenstruktur und Zündwinkelvorgabe 
3.6.6 Weitere Einsatzmöglichkeiten der Simulation im Applikationsprozess 245

3.6.7 Fazit

4 Getriebe

4.1 Optimierungsverfahren in der Antriebstechnik

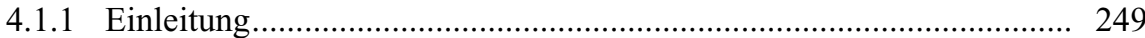

4.1.2 Gliederung der Optimierungsverfahren .......................................... 250

4.1.3 Topologieoptimierung: Begriffsklärung und Analogie ..................... 252

4.1.4 Prinzipielle Vorgehensweise bei einer Topologieoptimierung ........... 253

4.1.5 Grundlegende Erfahrungen bei der Topologieoptimierung eines

Differenzialdeckels ...................................................................... 254

4.1.6 Topografieoptimierung einer Ölwanne........................................... 267

4.1.7 Topologieoptimierung eines Getriebegehäuses ............................. 272

4.1.8 Optimierung eines Halteblechs ........................................................ 279

4.1.9 Einbindung in den Produktentwicklungsprozess .............................. 284

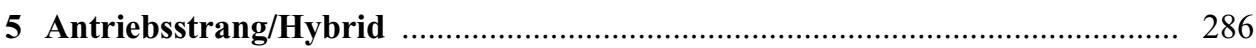

5.1 Modellbasierte Antriebsstrangentwicklung ........................................ 286

5.1.1 Einleitung .............................................................................. 286

5.1.2 Werkzeuge im modellbasierten Entwicklungsprozess ...................... 288

5.1.2.1 Systemmodell ............................................................... 288

5.1.2.2 Regelbasierte Modelltransformation .................................... 290

5.1.2.3 Physikalische und signalflussorientierte Modelle ............... 291

5.1.2.4 Optimierungswerkzeuge .................................................... 294

5.1.2.5 Modellbasierte Validierung ................................................. 294

5.1.3 Zusammenfassung ....................................................................... 296

5.2 Hybridfahrzeug in seiner Verkehrsumgebung .......................................... 298

5.2.1 Einleitung …......................................................................... 298

5.2.2 Simulationsumgebung .................................................................. 298

5.2.2.1 Längsdynamiksimulation ................................................ 299

5.2.3.2 Verkehrsszenarien ......................................................... 303

5.2.3 Simulationsergebnisse .............................................................. 305

5.2.3.1 Hybridantrieb .............................................................. 305

5.2.4 Zusammenfassung ....................................................................... 309

5.3 Einfluss des Strömungssiedens auf den kühlmittelseitigen Wärmeübergang in Verbrennungsmotoren ........................................................................ 310

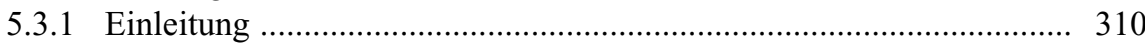

5.3 .2 Sieden ....................................................................................... 311

5.3.2.1 Beschreibung des Siede-Phänomens .................................. 311

5.3.2.2 Einflüsse .................................................................... 312

5.3.2.2.1 Kühlmittelzusammensetzung ............................ 313

5.3.2.2.2 Rauigkeit ........................................................ 314

5.3.2.2.3 Orientierung ..................................................... 314

5.3.2.2.4 Druckgradient ..................................................... 315

5.3.2.2.5 Vibration ............................................................ 315 
5.3.3 Modellbildung und Berechnungsmethodik ........................................ 316

5.3.3.1 Boiling-Departure-Liftoff (BDL)-Modell ............................. 316

5.3.3.2 Gekoppelte Berechnungsmethodik ……………................... 318

5.3.4 Anwendungsbeispiel ............................................................... 320

5.3.5 Zusammenfassung .......................................................................... 322

5.4 Simulation des NVH-Verhaltens im Antriebsstrang ...................................... 323

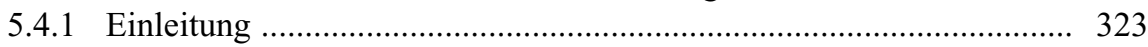

5.4.2 Modellkomplexität .................................................................... 324

5.4.3 Softwareprogramme und Modellierungsansätze ................................ 327

5.4.4 Untersuchung an einem Front-Querantriebsstrang .............................. 332

5.4.4.1 1D-Torsionsansatz ................................................................ 333

5.4.4.2 3D-MKS (Starkörper) ....................................................... 334

5.4.4.3 3D-MKS (flexible Körper) ……………………………....... 338

5.4.5 Zusammenfassung .......................................................................... 343

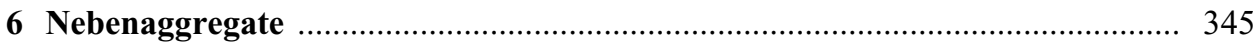

6.1 Simulation in der Produktentwicklung ....................................................... 345

6.1 .1 Einführung ……………….................................................. 345

6.1.2 Dynamikanalyse von Kühlmodulen ................................................ 345

6.1.3 Temperaturwechselbeanspruchung von Ladeluftkühlern ................... 347

6.1.4 Motorraumdurchströmung .............................................................. 348

6.1.5 Auslegung von Hybridquerträgern für Thermostrukturmodule ${ }^{\odot}$......... 350

6.1.6 Komfortbewertung mit dem virtuellen thermischen Dummy .............. 352

6.1.7 Dynamische Berechnung der Ladeluftkühlung mit BISS .................... 354

6.1.8 Füllungsoptimierung eines R744 Kältekreislaufs ................................ 355

6.2 Integrierte Virtuelle Gesamtfahrzeugsimulation ausgeführt am Beispiel des Thermischen Managements ................................................................. 358

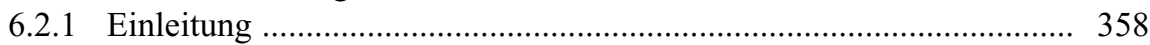

6.2.2 Motivation und Problemstellung ..................................................... 359

6.2.3 Technologien, Methoden und Werkzeuge für Integriertes Engineering 360

6.2.4 Rolle der Co-Simulation für das Thermische Management ................. 364

6.2.5 Design einer unabhängigen Co-Simulationsplattform-Erstellung eines Gesamtfahrzeugmodells am Beispiel des Thermischen Managements 366 6.2.5.1 Aufteilung des Gesamtsystems in Teilmodelle ..................... 367

6.2.5.2 Kopplung der Teilmodelle zu einem Gesamtmodell .............. 367

6.2.5.3 Synchronisierung der Teilmodelle innerhalb des

Gesamtmodells ................................................................ 369

6.2.6 Betrachtung der Energieströme innerhalb eines gekoppelten thermischen Gesamtfahrzeugmodells ............................ 372

6.2.7 Zusammenfassung und Ausblick ...................................................... 376

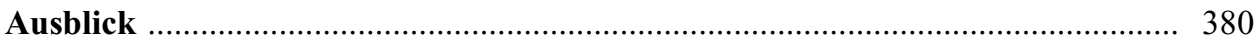

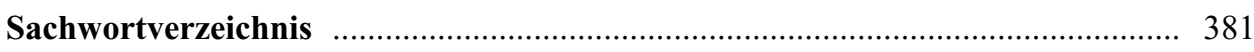




\section{Abkürzungen und Akronyme}

$\begin{array}{ll}\text { 2D } & \text { zweidimensional } \\ \text { 3D } & \text { dreidimensional } \\ \text { ADC } & \text { Analogue Digital Converter } \\ \text { AGR } & \text { Abgasrückführung } \\ \text { AR } & \text { Augmented Reality } \\ \text { AUTOSAR } & \text { AUTomotive Open System ARchitecture } \\ \text { BDL } & \text { Boiling Departure Liftoff } \\ \text { BISS } & \text { Behr integrierte Systemsimulation } \\ \text { BSA } & \text { Betriebsschwingungsanalyse } \\ \text { BSW } & \text { Basic Software } \\ \text { CAD } & \text { Computergestützte Konstruktion (Computer Aided Design) } \\ \text { CAE } & \text { Computergestütztes Engineering (Computer Aided Engineering) } \\ \text { CAx } & \text { Sammelbegriff für computergestützte Arbeitstechniken } \\ \text { CD } & \text { Compact Disc } \\ \text { CFD } & \text { Numerische 3D-Strömungssimulation (Computational Fluid Dynamics) } \\ \text { CPU } & \text { Central Processing Unit } \\ \text { CTA } & \text { Conformance Test Agency } \\ \text { CTS } & \text { Conformance Test Suite } \\ \text { CVT } & \text { Continuously Variable Transmission } \\ \text { DEM } & \text { Diagnostics Event Manager } \\ \text { DET } & \text { Development Error Tracer } \\ \text { DMU } & \text { Digitales Zusammenbau-Modell (Digital Mock-Up) } \\ \text { DoE } & \text { Design of Experiments (statistische Versuchsplanung) } \\ \text { DV-Technik } & \text { Datenverarbeitungstechniken } \\ \text { E/E } & \text { Electric/Electronics } \\ \text { ECU } & \text { Electronic Control Unit } \\ \text { ERP } & \text { Computergestützte Planung der Unternehmensressourcen } \\ & \text { (Enterprise Resource Planning) } \\ \text { FE } & \text { Finite Elemente } \\ \text { FEM } & \text { Finite-Elemente-Methode } \\ \text { FMEA } & \text { Fehlermöglichkeits- und Einflussanalyse } \\ \text { GIF } & \text { Graphic interchange Format - Komprimierendes Bilddateiformat } \\ \text { GJL } & \text { Gusseisen mit Lamellengraphit } \\ \text { GJV } & \text { Gusseisen mit Vermikulargraphit } \\ \text { HMD } & \text { Head-Mounted-Display } \\ \text { HMI } & \text { Human Machine Interface } \\ \text { HW } & \text { Hardware } \\ \text { I/O } & \text { Input/Output } \\ \text { IT } & \text { Informations-Technologie } \\ \text { KW } & \text { Kurbelwelle } \\ & \end{array}$




$\begin{array}{ll}\text { MARCO } & \text { Thermischer Dummy } \\ \text { MKS } & \text { Mehrkörpersimulation } \\ \text { MTBF } & \text { Mean Time Between Failures } \\ \text { NVH } & \text { Noise, Vibration and Harshness } \\ \text { PDM } & \text { Produkt-Daten-Management } \\ \text { PVS } & \text { Produktionsvorserie } \\ \text { RA } & \text { Risk Assessment } \\ \text { RFID } & \text { Radio Frequency Identification } \\ \text { RTE } & \text { Runtime Environment } \\ \text { SFE } & \text { Gesellschaft für Strukturanalyse in Forschung und Entwicklung mbH } \\ \text { SIMBAUK } & \text { Simulations-Baukasten } \\ \text { SOP } & \text { Start der Serienfertigung (Start of Production) } \\ \text { SW-C } & \text { Software Component } \\ \text { TSM } & \text { Thermostrukturmodul } \\ \text { UML } & \text { Unified Modeling Language } \\ \text { VB-Prozess } & \text { Versuchsbauprozess, dient der Erstellung von Prototypen } \\ \text { VFB } & \text { Virtual Functional Bus } \\ \text { ViP } & \text { Virtuelle Produktentwicklung } \\ \text { VR } & \text { Virtual Reality } \\ \text { VREP } & \text { virtueller Ergonomieprüfstand } \\ \text { XML } & \text { eXtensible Mark up Language } \\ \text { ZKG } & \text { Zylinder-Kurbel-Gehäuse } \\ & \end{array}$

